[6] R. Wang and J. Zhao, "Exponential stability analysis for discrete-time switched linear systems with time-delay," Int. J. Innov. Comput., Inform. Control, vol. 3, no. 6, pp. 1557-1564, 2007.

[7] L. Zhang, P. Shi, and E. Boukas, " $H_{\infty}$ output-feedback control for switched linear discrete-time systems with time-varying delays," Int. J. Control, vol. 80, no. 8, pp. 1354-1365, 2007.

[8] W. P. Dayawansa and C. F. Martin, "A converse Lyapunov theorem for a class of dynamical systems which undergo switching," IEEE Trans. Automat. Control, vol. 44, no. 4, pp. 751-760, Apr. 1999.

[9] A. S. Morse, Control Using Logic-Based Switching. Heidelberg, Germany: Springer-Verlag, 1997.

[10] M. Johansson and A. Rantzer, "Computation of piecewise quadratic Lyapunov functions for hybrid systems," IEEE Trans. Automat. Control, vol. 43, no. 4, pp. 555-559, Apr. 1998.

[11] J. Geromel and P. Colaneri, "Stability and stabilization of discrete time switched systems," Int. J. Control, vol. 79, no. 7, pp. 719-728, 2006.

[12] J. Lee and G. Dullerud, "Uniform stabilization of discrete-time switched and markovian jump linear systems," Automatica, vol. 42, no. 2, pp. 205-218, 2006.

[13] J. P. Hespanha and A. S. Morse, "Stability of switched systems with average dwell time," in Proc. 38th Conf. Decision Control, Phoenix, AZ, 1999, pp. 2655-2660.

[14] X. Sun, J. Zhao, and D. J. Hill, "Stability and $L_{2}$-gain analysis for switched delay systems: A delay-dependent method," Automatica, vol. 42, no. 10, pp. 1769-1774, 2006.

[15] L. Vu, D. Chatterjee, and D. Liberzon, "Input-to-state stability of switched systems and switching adaptive control," Automatica, vol. 43, no. 4, pp. 639-646, 2007.

[16] G. S. Zhai, B. Hu, K. Yasuda, and A. N. Michel, "Disturbance attenuation properties of time-controlled switched systems," J. Franklin Inst., vol. 338, no. 7, pp. 765-779, 2001.

[17] B. Lu and F. Wu, "Switching LPV control designs using multiple parameter-dependent lyapunov functions," Automatica, vol. 40, no. 11, pp. 1973-1980, 2004.

[18] S. Lim and J. P. How, "Modeling and $H_{\infty}$ control for switched linear parameter-varying missile autopilot," IEEE Trans. Control Syst. Technol., vol. 11, no. 6, pp. 830-838, Nov. 2003.

[19] B. Lu, F. Wu, and S. K, "Switching LPV control of an F-16 aircraft via controller state reset," IEEE Trans. Control Syst. Technol., vol. 14, no. 2, pp. 267-277, Mar. 2006.

[20] H. Nishimura and K. Funaki, "Motion control of three-link brachiation robot by using final-state control with error learning," IEEE/ASME Trans. Mechatron., vol. 3, no. 2, pp. 120-128, Jun. 1998.

[21] G. Millerioux, L. Rosier, G. Bloch, and J. Daafouz, "Bounded state reconstruction error for LPV systems with estimated parameters," IEEE Trans. Automat. Control, vol. 49, no. 8, pp. 1385-1389, Aug. 2004.

[22] P. Pellanda, P. Apkarian, and H. Tuan, "Missile autopilot design via a multi-channel LFT/LPV control method," Int. J. Robust Nonlin. Control, vol. 12, no. 1, pp. 1-20, 2002.

[23] J. Gao and H. Budman, "Design of robust gain-scheduled PI controllers for nonlinear processes," J. Process Control, vol. 15, no. 7, pp. 807-817, 2005.

[24] W.-Y. Yan and J. Lam, "An approximate approach to $H^{2}$ optimal model reduction," IEEE Trans. Automat. Control, vol. 44, no. 7, pp. 1341-1358, Jul. 1999.

[25] K. Zhou, "Frequency weighted $L_{\infty}$ norm and optimal Hankel norm model reduction," IEEE Trans. Automat. Control, vol. 40, no. 10, pp. 1687-1699, Oct. 1995.

[26] K. Zhou, J. C. Doyle, and K. Glover, Robust and Optimal Control. Upper Saddle River, NJ: Prentice-Hall, 1996.

[27] Y. Ebihara and T. Hagiwara, "On $H_{\infty}$ model reduction using LMIs," IEEE Trans. Automat. Control, vol. 49, no. 7, pp. 1187-1191, Jul. 2004.

[28] H. Gao, J. Lam, C. Wang, and S. Xu, " $H_{\infty}$ model reduciton for discrete time-delay systems: Delay-independent and dependent approaches," Int. J. Control, vol. 77, no. 4, pp. 321-335, 2004.

[29] S. Xu and J. Lam, " $H_{\infty}$ model reduction for discrete-time singular systems," Syst. Control Lett., vol. 48, pp. 121-133, 2002.

[30] C. Gong and B. Su, "Robust $L_{2}-L_{\infty}$ filtering of convex polyhedral uncertain time-delay fuzzy systems," Int. J. Innov. Comput., Inform. Control, vol. 4, no. 4, pp. 793-802, 2008.

[31] L. Zhang, P. Shi, E. Boukas, and C. Wang, "Robust $l_{2}-l_{\infty}$ filtering for switched linear discrete time-delay systems with polytopic uncertainties," IET Control Theory Appl., vol. 1, no. 3, pp. 722-730, 2007.
[32] L. Zhang, E. Boukas, and P. Shi, "Exponential $H_{\infty}$ filtering for uncertain discrete-time switched linear systems with average dwell time: A $\mu$-dependent approach," Int. J. Robust Nonlin. Control, vol. 18, no. 11, pp. 1188-1207, 2008.

[33] P. Apkarian and R. J. Adams, "Advanced gain-scheduling techniques for uncertain systems processes," IEEE Trans. Control Syst. Technol., vol. 6, no. 1, pp. 21-32, Jan. 1998.

[34] F. Wu and K. Grigoriadis, "LPV systems with parameter-varying time delays: Analysis and control," Automatica, vol. 37, no. 2, pp. 221-229, 2001.

\section{Filtering for Nonlinear Genetic Regulatory Networks With Stochastic Disturbances}

\author{
Zidong Wang, James Lam, Guoliang Wei, Karl Fraser, and \\ Xiaohui Liu
}

\begin{abstract}
In this paper, the filtering problem is investigated for nonlinear genetic regulatory networks with stochastic disturbances and time delays, where the nonlinear function describing the feedback regulation is assumed to satisfy the sector condition, the stochastic perturbation is in the form of a scalar Brownian motion, and the time delays exist in both the translation process and the feedback regulation process. The purpose of the addressed filtering problem is to estimate the true concentrations of the mRNA and protein. Specifically, we are interested in designing a linear filter such that, in the presence of time delays, stochastic disturbances as well as sector nonlinearities, the filtering dynamics of state estimation for the stochastic genetic regulatory network is exponentially mean square stable with a prescribed decay rate lower bound $\beta$. By using the linear matrix inequality (LMI) technique, sufficient conditions are first derived for ensuring the desired filtering performance for the gene regulatory model, and the filter gain is then characterized in terms of the solution to an LMI, which can be easily solved by using standard software packages. A simulation example is exploited in order to illustrate the effectiveness of the proposed design procedures.
\end{abstract}

Index Terms-Decay rate, gene expression, genetic regulatory network, stochastic disturbance, time-delay.

\section{INTRODUCTION}

It is well known that the encoded information contained in genes is necessary for the organism to develop within a changing external en-

Manuscript received August 27, 2007; revised December 12, 2007. Current version published November 05,2008 . This work was supported in part by the Biotechnology and Biological Sciences Research Council (BBSRC) of the U.K. under Grants BB/C506264/1 and 100/EGM17735, the Engineering and Physical Sciences Research Council (EPSRC) of the U.K. under Grants GR/S27658/01 and EP/C524586/1, an International Joint Project sponsored by the Royal Society of the U.K., the Nuffield Foundation of the U.K. under Grant NAL/00630/G, and the Alexander von Humboldt Foundation of Germany. Recommended by Associate Editor B. Ninness.

Z. Wang, K. Fraser, and X. Liu are with the Department of Information Systems and Computing, Brunel University, Middlesex UB8 3PH, U.K. (e-mail: zidong.wang@brunel.ac.uk).

J. Lam is with the Department of Mechanical Engineering, The University of Hong Kong, Hong Kong, China.

G. Wei is with the School of Information Science and Technology, Donghua University, Shanghai 200051, China.

Color versions of one or more of the figures in this paper are available online at http://ieeexplore.ieee.org.

Digital Object Identifier 10.1109/TAC.2008.2007862 
vironment. Genetic regulatory networks are the mechanisms that have evolved to regulate the expression of genes, where the expression level of a gene is regulated negatively or positively by its own production (protein). DNA microarray technology [25] has made it possible to measure gene expression levels on a genomic scale, and has therefore been extensively applied to gene transcription analysis. Theoretical analysis and experimental investigation on genetic regulatory networks (GRNs) have quickly become an attractive area of research in the biological and biomedical sciences, and received great attention over past decade [5], [7], [9], [13], [17]-[20], [26], [33], [36].

Recently, there has been much interest to reconstruct models for GRNs, for example, Boolean network models [14], linear differential equation models [4], [8], [15], [33], and a single negative feedback loop network [9]. In biological systems or artificial genetic networks, time delays exist due primarily to the slow processes of transcription, translation, translocation as well as the finite switching speed of amplifiers. It has been pointed out in [5], [28], [29] that delays may play an important role in dynamics of genetic networks, and mathematical models without addressing the delay effects may even provide wrong predictions of the mRNA and protein concentrations [28], [29]. It has also been shown in [23], by mathematically modelling recent data, that the observed oscillatory expression and activity of three proteins are most likely driven by transcriptional delays. Very recently, the GRN with SUM regulatory logic has been first introduced in [18] with the asymptotic stability being discussed, and then subsequent research on stochastic stability and stochastic synchronization has been carried out in [19], [20]. Delayed GRNs have been addressed in [26] where the time delays are assumed to be time-varying and belong to given intervals.

On the other hand, when modeling GRNs, the stochastic noise (fluctuations) in real-world gene expression data is of great importance [1], [6], [19], [20], [30], [33]. In general, the stochastic noise arises in gene expression in one of two ways, namely, internal noise and external noise. The internal random fluctuations in genetic networks are inevitable as chemical reactions are probabilistic [24] and the external noise originates in the random variation of one or more of the externally set control parameters [16]. Recently, in [3], a stochastic nonlinear dynamic model has been developed for GRNs under intrinsic fluctuation and extrinsic noise, and a method has been proposed to determine the robust stability under intrinsic fluctuations and identify the genes that are significantly affected by extrinsic noises.

In practice, for the ultimate goal of identifying genes of interest and designing drugs, biologists would be interested in knowing the steady-state values of the actual network states, that is, the concentrations of the mRNA and protein. Unfortunately, due to the inherent state delay and state-dependent noises, the actual network measurements are far from the true network states, and any subsequent analysis based only on the network measurements would probably have little value in application. This gives rise to the following 'filtering' research issue: given a gene regulatory network that contains both transmission delays and intrinsic fluctuations, how to estimate the network states such that the estimation error could exponentially converge to zero in the mean square sense? Such a filtering issue has been addressed in [35] for a linear GRN with stochastic disturbances in terms of the variance-constrained index, where the time delay and regulation nonlinearities have been ignored. It should be pointed out that, up to now, most existing literature has dealt with the dynamical behaviors of various GRNs, but the important filtering problem for GRNs has been largely overlooked despite its practical significance. Although the filtering problem has been extensively studied in the control and signal processing communities (see [11], [12], [31], [32], [34] and references therein), the filtering problem for GRNs of specific structures still remains challenging, which constitutes the main focus of this paper.
This paper is concerned with the filtering problem for a class of nonlinear genetic regulatory networks with state-dependent stochastic disturbances as well as state delays. The feedback regulation is described by a sector-like nonlinear function, the stochastic perturbation is a scalar Brownian motion, and the time delays enter into both the translation process and the feedback regulation process. We aim to estimate the true concentrations of the mRNA and protein by designing a linear filter with guaranteed exponential stability of the filtering augmented systems. By using the linear matrix inequality (LMI) technique, sufficient conditions are first derived for ensuring the exponentially mean square stable with a prescribed decay rate lower bound $\beta$ for the gene regulatory model, and then the filter gain is characterized in terms of the solution to an LMI, which can be easily solved by using available software packages. A simulation example is illustrated for a gene expression model.

Notation: Throughout this paper, $\mathbb{R}^{n}$ denotes the $n$ dimensional Euclidean space. $L_{2}[0, \infty)$ is the space of square-integrable vector functions over $[0, \infty) ;|\cdot|$ refers to the Euclidean norm in $\mathbb{R}^{n}$, and $\|\cdot\|_{2}$ stands for the usual $L_{2}[0, \infty)$ norm. $I$ denotes the identity matrix of compatible dimension. The notation $X \geq Y$ (respectively, $X>Y$ ) where $X$ and $Y$ are symmetric matrices, means that $X-Y$ is positive semi-definite (respectively, positive definite). For a matrix $M, M^{T}$ represents its transpose and $\|M\|$ denotes its spectral norm. When $M$ is real symmetric, $\lambda_{\max }(M)$ (respectively, $\lambda_{\min }(M)$ ) stands for its maximum (respectively, minimum) eigenvalue. $\left(\Omega, \mathcal{F},\left\{\mathcal{F}_{t}\right\}_{t \in \mathbb{R}}, \mathcal{P}\right)$ is a complete probability space with a filtration $\left\{\mathcal{F}_{t}\right\}_{t \in \mathbb{R}}$ satisfying the usual conditions (i.e., the filtration contains all $P$-null sets and is right continuous). $\mathbb{E}\{x\}$ stands for the expectation of stochastic variable $x$. The shorthand $\operatorname{diag}\left\{M_{1}, \ldots, M_{n}\right\}$ denotes a block diagonal matrix with diagonal blocks $M_{1}, \ldots, M_{n}$ and the notation $\operatorname{diag}_{q}\{\star\}$ is employed to stand for $\operatorname{diag}\{\star, \ldots, \star\}$ with $q$ blocks of $\star$. In symmetric block matrices, the symbol $*$ is used as an ellipsis for terms induced by symmetry. Matrices, if not explicitly stated, are assumed to have compatible dimensions.

\section{PROBLEM Formulation}

In this paper, we consider the following nonlinear time-delay genetic regulatory networks [5], [18], [26] with state-dependent stochastic disturbances:

$$
(\Sigma):\left\{\begin{array}{l}
d x_{m}(t)=\left[-A_{1} x_{m}(t)+B g\left(x_{p}\left(t-\tau_{1}\right)\right)\right] d t \\
\quad+E x_{m}(t) d \omega_{1}(t) \\
d x_{p}(t)=\left[-A_{2} x_{p}(t)+D x_{m}\left(t-\tau_{2}\right)\right] d t \\
\quad+F x_{p}(t) d \omega_{2}(t) \\
y_{m}(t)=C_{1} x_{m}(t) \\
y_{p}(t)=C_{2} x_{p}(t) \\
x_{m}(t)=\phi_{m}(t), x_{p}(t)=\phi_{p}(t), \forall t \in[-2 \tau, 0]
\end{array}\right.
$$

where $x_{m}(t)=\left[\begin{array}{llll}x_{m 1}(t) & x_{m 2}(t) & \cdots & x_{m n}(t)\end{array}\right]^{T} \in \mathbb{R}^{n}, x_{p}(t)=$ $\left[x_{p 1}(t) x_{p 2}(t) \cdots x_{p n}(t)\right]^{T} \in \mathbb{R}^{n} ; x_{m i}(t)$ and $x_{p i}(t)(i=1, \ldots, n)$ denote the concentrations of mRNA and protein of the $i$ th node at time $t$, respectively; $y_{m}(t)=\left[y_{m 1}(t) y_{m 2}(t) \cdots y_{m r}(t)\right]^{T} \in \mathbb{R}^{r}, y_{p}(t)=$ $\left[y_{p 1}(t) y_{p 2}(t) \cdots y_{p r}(t)\right]^{T} \in \mathbb{R}^{r} ; y_{m j}(t)$ and $y_{p j}(t)(j=1, \ldots, r)$ represent the expression levels of mRNA and protein of the $j$ th node at time $t$, respectively; $\phi_{m}(t), \phi_{p}(t)$ are the initial functions of $x_{m}(t)$ and $x_{p}(t)$, respectively; and $g\left(x_{p}\left(t-\tau_{1}\right)\right)=\left[g_{1}\left(x_{p 1}\left(t-\tau_{1}\right)\right) g_{2}\left(x_{p 2}(t-\right.\right.$ $\left.\left.\left.\tau_{1}\right)\right) \cdots g_{n}\left(x_{p n}\left(t-\tau_{1}\right)\right)\right]^{T} \in \mathbb{R}^{n}$ with the function $g_{i}(\cdot)$ representing the feedback regulation of the protein on the transcription, which is generally a nonlinear function but has a form of monotonicity with each variable [1]. Both $\omega_{1}(t)$ and $\omega_{2}(t)$ are scalar Brownian motions with zero mean value and unit variance, and they are mutually uncorrelated. The constants $\tau_{1}>0, \tau_{2}>0$ denote, respectively, the translation 
delay and the feedback regulation delay. Let $\tau=\max \left\{\tau_{1}, \tau_{2}\right\}$. The matrix $B=\left(b_{i j}\right) \in \mathbb{R}^{n \times n}$ is defined as follows:

$$
b_{i j} \begin{cases}>0, & \text { if transcription factor } j \text { is an } \\ & \text { activator of gene } i ; \\ <0, & \text { if there is no link from node } j \text { to } i \\ <0, & \text { if transcription factor } j \text { is a } \\ & \text { repressor of gene } i .\end{cases}
$$

The matrices $A_{1}=\operatorname{diag}\left\{a_{11}, a_{12}, \ldots, a_{1 n}\right\}, A_{2}=$ $\operatorname{diag}\left\{c_{11}, c_{12}, \ldots, c_{1 n}\right\}$ and $D=\operatorname{diag}\left\{d_{1}, d_{2}, \ldots, d_{n}\right\}$ are diagonal matrices with $a_{1 i}, c_{1 i}, d_{i}(i=1, \ldots, n)$ being the rate of degradation of mRNA, the rate of degradation of protein and the translation rate of the $i$ th node, respectively. $A_{1}, A_{2}, B, C_{1}, C_{2}, D$, $E$ and $F$ are all constant matrices with appropriate dimensions.

Assumption 1: The function $g_{i}(\cdot)$ satisfies the following sector condition

$$
\begin{aligned}
0 \leq f_{i}\left(x_{i}\right):=\frac{g_{i}\left(x_{i}\right)}{x_{i}} \leq k_{i}, \forall x_{i} & \in \mathbb{R}, x_{i} \neq 0 ; \text { and } \\
f_{i}(0) & =0, g_{i}(0)=0, i=1, \ldots, n,
\end{aligned}
$$

which is equivalent to

$$
g^{T}(x)(g(x)-K x) \leq 0
$$

where $K=\operatorname{diag}\left\{k_{1}, k_{2}, \ldots, k_{n}\right\}>0$.

Remark 1: By Assumption 1, the GRN (1) can be regarded as a kind of Lur'e systems with stochastic disturbances, in which the fruitful Lur'e system method in control theory [17] could be applied. Notice that the sector-like nonlinear function $g_{i}(\cdot)$ has been used to model the structure and regulation mechanism of the genetic networks in many references. For example, as a monotonic increasing or decreasing regulatory function, $g_{i}(\cdot)$ is usually of the Michaelis-Menten or Hill form [17], which can easily be transformed to a nonlinear function satisfying the sector condition.

Remark 2: For presentation simplicity, the noise intensity functions in model (1) are assumed to be linear. We point out that our main results can be easily extended to the case when the noise intensity functions $h_{1}\left(x_{m}(t), x_{m}\left(t-\tau_{2}\right)\right)$ and $h_{2}\left(x_{p}(t), x_{p}\left(t-\tau_{1}\right)\right)$ are nonlinear and satisfy the following bounding conditions

$$
\begin{aligned}
& h_{1}^{T}\left(x_{m}(t), x_{m}\left(t-\tau_{2}\right)\right) h_{1}\left(x_{m}(t), x_{m}\left(t-\tau_{2}\right)\right) \\
& \quad \leq\left\|E_{1} x_{m}(t)\right\|^{2}+\left\|E_{2} x_{m}\left(t-\tau_{2}\right)\right\|^{2} \\
& h_{2}^{T}\left(x_{p}(t), x_{p}\left(t-\tau_{1}\right)\right) h_{2}\left(x_{p}(t), x_{p}\left(t-\tau_{1}\right)\right) \\
& \quad \leq\left\|F_{1} x_{p}(t)\right\|^{2}+\left\|F_{2} x_{p}\left(t-\tau_{1}\right)\right\|^{2},
\end{aligned}
$$

where $E_{1}, E_{2}, F_{1}, F_{2}$ are known constant matrices.

The main aim of this paper is to estimate the concentrations of mRNA and protein through their expression level. The linear filter adopted is of the form

$$
\left(\Sigma_{f}\right):\left\{\begin{array}{l}
d \hat{x}_{m}(t)=\hat{A} \hat{x}_{m}(t) d t+\hat{B} y_{m}(t) d t \\
d \hat{x}_{p}(t)=\hat{C} \hat{x}_{p}(t) d t+\hat{D} y_{p}(t) d t \\
\hat{x}_{m}(t)=\psi_{m}(t), \hat{x}_{p}(t)=\psi_{p}(t), \forall t \in[-2 \tau, 0]
\end{array}\right.
$$

where $\hat{x}_{m}(t) \in \mathbb{R}^{n}$ and $\hat{x}_{p}(t) \in \mathbb{R}^{n}$ are the estimates for $x_{m}(t)$ and $x_{p}(t)$, respectively; $\psi_{m}(t)$ and $\psi_{p}(t)$ are the initial functions of $\hat{x}_{m}(t)$ and $\hat{x}_{p}(t)$, respectively; and $\hat{A}, \hat{B}, \hat{C}$ and $\hat{D}$ are filter parameters to be determined.
By defining

$$
\begin{aligned}
\bar{x}_{m}(t) & :=\left[\begin{array}{l}
x_{m}(t) \\
\hat{x}_{m}(t)
\end{array}\right], \quad \bar{x}_{p}(t):=\left[\begin{array}{l}
x_{p}(t) \\
\hat{x}_{p}(t)
\end{array}\right] \\
\bar{x}_{m \tau_{2}} & :=\left[\begin{array}{l}
x_{m}\left(t-\tau_{2}\right) \\
\hat{x}_{m}\left(t-\tau_{2}\right)
\end{array}\right], \quad \bar{x}_{p \tau_{1}}:=\left[\begin{array}{l}
x_{p}\left(t-\tau_{1}\right) \\
\hat{x}_{p}\left(t-\tau_{1}\right)
\end{array}\right],
\end{aligned}
$$

and combining $(\Sigma)$ and $\left(\Sigma_{f}\right)$, we obtain the augmented filtering dynamics as follows:

$$
\left(\Sigma_{e}\right):\left\{\begin{array}{l}
d \bar{x}_{m}(t)=\bar{A} \bar{x}_{m}(t) d t+\bar{B} g\left(Z \bar{x}_{p \tau_{1}}\right) d t+\bar{E} Z \bar{x}_{m}(t) d \omega_{1}(t) \\
d \bar{x}_{p}(t)=\bar{C} \bar{x}_{p}(t) d t+\bar{D} Z \bar{x}_{m \tau_{2}} d t+\bar{F} Z \bar{x}_{p}(t) d \omega_{2}(t)
\end{array}\right.
$$

where

$$
\begin{array}{ll}
\bar{A}=\left[\begin{array}{ll}
-A_{1} & 0 \\
\hat{B} C_{1} & \hat{A}
\end{array}\right], & \bar{B}=\left[\begin{array}{l}
B \\
0
\end{array}\right], \quad \bar{E}=\left[\begin{array}{l}
E \\
0
\end{array}\right] \\
\bar{C}=\left[\begin{array}{ll}
-A_{2} & 0 \\
\hat{D} C_{2} & \hat{C}
\end{array}\right], & \bar{D}=\left[\begin{array}{c}
D \\
0
\end{array}\right], \quad \bar{F}=\left[\begin{array}{c}
F \\
0
\end{array}\right], Z=\left[\begin{array}{ll}
I & 0
\end{array}\right] .
\end{array}
$$

For presentation convenience, we let

$$
\left\{\begin{array}{l}
\xi_{m}(t)=\bar{A} \bar{x}_{m}(t)+\bar{B} g\left(Z \bar{x}_{p \tau_{1}}\right) \\
\xi_{p}(t)=\bar{C} \bar{x}_{p}(t)+\bar{D} Z \bar{x}_{m \tau_{2}}
\end{array}\right.
$$

and then $\left(\Sigma_{e}\right)$ in (6) can be rewritten as

$$
\left\{\begin{array}{l}
d \bar{x}_{m}(t)=\xi_{m}(t) d t+\bar{E} Z \bar{x}_{m}(t) d \omega_{1}(t) \\
d \bar{x}_{p}(t)=\xi_{p}(t) d t+\bar{F} Z \bar{x}_{p}(t) d \omega_{2}(t) .
\end{array}\right.
$$

Before formulating the problem to be investigated, we first introduce the following stability concept for the augmented system (6).

Definition 1: System (6) is said to be exponentially mean square stable if there exist scalars $\alpha>0$ and $\beta>0$ such that

$$
\mathbb{E}|\bar{x}(t, \rho)|^{2} \leq \alpha e^{-\beta t} \sup _{-2 \tau \leq t \leq 0} \mathbb{E}|\rho(t)|^{2}, \quad \forall \rho(t) \in \mathbb{R}^{4 n \times n}
$$

or, equivalently,

$$
\lim _{t \rightarrow \infty} \sup \frac{1}{t} \log \left(\mathbb{E}|\bar{x}(t, \rho)|^{2}\right) \leq-\beta,
$$

where $\quad \bar{x}(t) \quad:=\quad\left[\bar{x}_{m}^{T}(t) \bar{x}_{p}^{T}(t)\right]^{T} \quad$ and $\quad \rho(t) \quad:=$ $\left[\phi_{m}^{T}(t) \psi_{m}^{T}(t) \phi_{p}^{T}(t) \psi_{p}^{T}(t)\right]^{T}$ is the initial function of $\bar{x}(t)$.

The purpose of this paper is to design a desired filter of the form (5) for the system $(\Sigma)$ in (1) such that, for all admissible time delays, nonlinearities and stochastic disturbances, the augmented system (6) is exponentially mean square stable.

\section{MAIN RESULTS}

\section{A. Filter Analysis}

First, we give the following lemmas which will be used in the proofs of our main results in this paper.

Lemma 1: [2] (Schur Complement) Given constant matrices $\Sigma_{1}, \Sigma_{2}, \Sigma_{3}$ where $\Sigma_{1}=\Sigma_{1}^{T}$ and $0<\Sigma_{2}=\Sigma_{2}^{T}$. Then $\Sigma_{1}+\Sigma_{3}^{T} \Sigma_{2}^{-1} \Sigma_{3}<0$ if and only if

$$
\left[\begin{array}{cc}
\Sigma_{1} & \Sigma_{3}^{T} \\
\Sigma_{3} & -\Sigma_{2}
\end{array}\right]<0 \quad \text { or } \quad\left[\begin{array}{cc}
-\Sigma_{2} & \Sigma_{3} \\
\Sigma_{3}^{T} & \Sigma_{1}
\end{array}\right]<0
$$

Lemma 2: Let $x \in \mathbb{R}^{n}, y \in \mathbb{R}^{n}$ and matrix $Q>0$. Then, we have $x^{T} y+y^{T} x \leq x^{T} Q^{-1} x+y^{T} Q y$. 


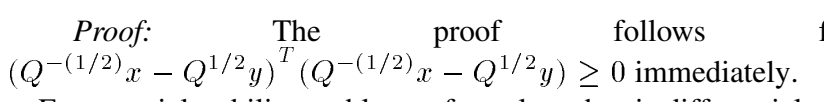

Exponential stability problems of usual stochastic differential equations have been considered in [22] through the construction of an appropriate Lyapunov function. In the following theorem, a delay-dependent LMI method is used to deal with the exponentially mean square stable problem for the augmented nonlinear stochastic genetic regulatory model (6), and a sufficient condition is derived that ensures the solvability of the filtering problem.

Theorem 1: Consider system (6) with given filter parameters. For a prescribed constant $\beta>0$, if there exist matrices $P_{i}>0$, diagonal matrices $Q_{i}>0, R_{i}>0, S_{i}>0$ and scalars $\varepsilon_{i}>0(i=1,2)$ such that the linear matrix inequality, shown at bottom of the page, holds, where

$$
\begin{aligned}
& \Omega_{1}:=\bar{A}^{T} P_{1}+P_{1} \bar{A}+Z^{T} S_{2} Z+\beta P_{1} \\
& \Omega_{2}:=\bar{C}^{T} P_{2}+P_{2} \bar{C}+Z^{T} K S_{1} K Z+\beta P_{2} \\
& \mathcal{U}_{1}:=\left[\begin{array}{lll}
P_{1} \bar{B} \tau_{1} P_{1} \bar{B} K P_{1} \bar{B} K \varepsilon_{1} Z^{T} E^{T} Z^{T} Q_{1}
\end{array}\right] \\
& \mathcal{U}_{2}:=\left[\begin{array}{lll}
P_{2} \bar{D} & \tau_{2} P_{2} \bar{D} P_{2} \bar{D} \varepsilon_{2} Z^{T} F^{T} Z^{T} Q_{2}
\end{array}\right] \\
& \mathcal{V}_{1}:=\left[\begin{array}{lll}
S_{1} & \tau_{1} R_{2} \varepsilon_{2} I \frac{\varepsilon_{1} \beta}{e^{\beta \tau_{2}}-1} I e^{-\beta \tau_{2}} Q_{1}
\end{array}\right] \\
& \mathcal{V}_{2}:=\left[\begin{array}{lll}
S_{2} & \tau_{2} R_{1} \varepsilon_{1} I \frac{\varepsilon_{2} \beta}{e^{\beta \tau_{1}}-1} I e^{-\beta \tau_{1}} Q_{2}
\end{array}\right]
\end{aligned}
$$

then the augmented system (6) is exponentially mean square stable.

Proof: Recalling the Newton-Leibniz formula and (8), we can write

$$
\left\{\begin{array}{c}
\bar{x}_{m}\left(t-\tau_{2}\right)=\bar{x}_{m}(t)-\int_{t-\tau_{2}}^{t} d \bar{x}_{m}(t)=\bar{x}_{m}(t) \\
-\int_{t-\tau_{2}}^{t} \xi_{m}(s) d s-\int_{t-\tau_{2}}^{t} \bar{E} Z \bar{x}_{m}(s) d \omega_{1}(s) \\
\bar{x}_{p}\left(t-\tau_{1}\right)=\bar{x}_{p}(t)-\int_{t-\tau_{1}}^{t} d \bar{x}_{p}(t)=\bar{x}_{p}(t) \\
-\int_{t-\tau_{1}}^{t} \xi_{p}(s) d s-\int_{t-\tau_{1}}^{t} \bar{F} Z \bar{x}_{p}(s) d \omega_{2}(s) .
\end{array}\right.
$$

It is easy to know from (3) and (12) that (6) is equivalent to the following system:

$$
\left\{\begin{array}{c}
d \bar{x}_{m}(t)=\bar{A} \bar{x}_{m}(t) d t+\bar{B} f_{\tau_{1}} Z\left[\bar{x}_{p}(t)-\int_{t-\tau_{1}}^{t} \xi_{p}(s) d s\right. \\
\left.-\int_{t-\tau_{1}}^{t} \bar{F} Z \bar{x}_{p}(s) d \omega_{2}(s)\right] d t+\bar{E} Z \bar{x}_{m}(t) d \omega_{1}(t) \\
d \bar{x}_{p}(t)=\bar{C} \bar{x}_{p}(t) d t+\bar{D} Z\left[\bar{x}_{m}(t)-\int_{t-\tau_{2}}^{t} \xi_{m}(s) d s\right. \\
\left.-\int_{t-\tau_{2}}^{t} \bar{E} Z \bar{x}_{m}(s) d \omega_{1}(s)\right] d t+\bar{F} Z \bar{x}_{p}(t) d \omega_{2}(t),
\end{array}\right.
$$

where

$$
\begin{array}{r}
f_{\tau_{1}}=\operatorname{diag}\left\{f_{1}\left(\bar{x}_{p 1}\left(t-\tau_{1}\right)\right), f_{2}\left(\bar{x}_{p 2}\left(t-\tau_{1}\right)\right), \ldots,\right. \\
\left.f_{n}\left(\bar{x}_{p n}\left(t-\tau_{1}\right)\right)\right\}
\end{array}
$$

with $\bar{x}_{p i}\left(t-\tau_{1}\right)(i=1,2, \ldots, n)$ denoting the $i$ th element of the vector $\bar{x}_{p \tau_{1}}$, and $f_{i}(x)$ is defined in (3). Hence, we only need to confirm that the system (13) is exponentially mean square stable.

In order to show that system (13) is exponentially mean square stable under condition (10), we define the following Lyapunov-Krasovskii functional candidate for system (13):

$$
\begin{aligned}
V(t)= & e^{\beta t} \bar{x}_{m}^{T}(t) P_{1} \bar{x}_{m}(t)+e^{\beta t} \bar{x}_{p}^{T}(t) P_{2} \bar{x}_{p}(t) \\
& +\int_{t-\tau_{2}}^{t} e^{\beta\left(s+\tau_{2}\right)} \bar{x}_{m}^{T}(s) Z^{T} Q_{1} Z \bar{x}_{m}(s) d s \\
& +\int_{t-\tau_{1}}^{t} e^{\beta\left(s+\tau_{1}\right)} \bar{x}_{p}^{T}(s) Z^{T} Q_{2} Z \bar{x}_{p}(s) d s \\
& +\int_{-\tau_{2}}^{0} \int_{t+s}^{t} e^{\beta(\theta-s)} \xi_{m}^{T}(\theta) Z^{T} R_{1} Z \xi_{m}(\theta) d \theta d s \\
& +\int_{-\tau_{1}}^{0} \int_{t+s}^{t} e^{\beta(\theta-s)} \xi_{p}^{T}(\theta) Z^{T} R_{2} Z \xi_{p}(\theta) d \theta d s \\
& +\varepsilon_{1} \int_{-\tau_{2}}^{t} \int_{t+s}^{t} e^{\beta(\theta-s)} \bar{x}_{m}^{T}(\theta) Z^{T} E^{T} E Z \bar{x}_{m}(\theta) d \theta d s \\
& +\varepsilon_{2} \int_{-\tau_{1}}^{0} \int_{t+s}^{t} e^{\beta(\theta-s)} \bar{x}_{p}^{T}(\theta) Z^{T} F^{T} F Z \bar{x}_{p}(\theta) d \theta d s .
\end{aligned}
$$

By Itô's differential formula [21], we have

$$
\begin{array}{r}
d V(t)=\mathcal{L} V(t) d t+2 e^{\beta t} \bar{x}_{m}^{T}(t) P_{1} \bar{E} Z \bar{x}_{m}(t) d \omega_{1}(t) \\
+2 e^{\beta t} \bar{x}_{p}^{T}(t) P_{2} \bar{F} Z \bar{x}_{p}(t) d \omega_{2}(t),
\end{array}
$$

where

$$
\begin{aligned}
\mathcal{L} V(t)= & \beta e^{\beta t} \bar{x}_{m}^{T}(t) P_{1} \bar{x}_{m}(t) \\
& +e^{\beta t} \bar{x}_{m}^{T}(t)\left(\bar{A}^{T} P_{1}+P_{1} \bar{A}\right) \bar{x}_{m}^{T}(t) \\
& +e^{\beta t} \bar{x}_{m}^{T}(t) Z^{T} \bar{E}^{T} P_{1} \bar{E} Z \bar{x}_{m}(t)
\end{aligned}
$$

$\left[\begin{array}{ccccccccccc}\Omega_{1} & 0 & 0 & 0 & 0 & \bar{A}^{T} Z^{T} R_{1} & 0 & \mathcal{U}_{1} & 0 & Z^{T} \bar{E}^{T} P_{1} & 0 \\ * & \Omega_{2} & 0 & 0 & 0 & 0 & \bar{C}^{T} Z^{T} R_{2} & 0 & \mathcal{U}_{2} & 0 & Z^{T} \bar{F}^{T} P_{2} \\ * & * & -Q_{1} & 0 & 0 & 0 & \bar{D}^{T} Z^{T} R_{2} & 0 & 0 & 0 & 0 \\ * & * & * & -Q_{2} & K & 0 & 0 & 0 & 0 & 0 & 0 \\ * & * & * & * & -2 I & \bar{B}^{T} Z^{T} R_{1} & 0 & 0 & 0 & 0 & 0 \\ * & * & * & * & * & -\frac{\beta}{e^{\beta \tau}-1} R_{1} & 0 & 0 & 0 & 0 & 0 \\ * & * & * & * & * & * & -\frac{\beta}{e^{\beta} \tau_{1}-1} R_{2} & 0 & 0 & 0 & 0 \\ * & * & * & * & * & * & * & -\mathcal{V}_{1} & 0 & 0 & 0 \\ * & * & * & * & * & * & * & * & -\mathcal{V}_{2} & 0 & 0 \\ * & * & * & * & * & * & * & * & * & -P_{1} & 0 \\ * & * & * & * & * & * & * & * & * & * & -P_{2}\end{array}\right]<0$




$$
\begin{aligned}
& +2 e^{\beta t} \bar{x}_{m}^{T}(t) P_{1} \bar{B} f_{\tau_{1}} Z \bar{x}_{p}(t)-2 e^{\beta t} \bar{x}_{m}^{T}(t) P_{1} \bar{B} f_{\tau_{1}} Z \\
& \times\left[\int_{t-\tau_{1}}^{t} \xi_{p}(s) d s+\int_{t-\tau_{1}}^{t} \bar{F} Z \bar{x}_{p}(s) d \omega_{2}(s)\right] \\
& +\beta e^{\beta t} \bar{x}_{p}^{T}(t) P_{2} \bar{x}_{p}(t)+e^{\beta t} \bar{x}_{p}^{T}(t)\left(\bar{C}^{T} P_{2}+P_{2} \bar{C}\right) \bar{x}_{p}(t) \\
& +e^{\beta t} \bar{x}_{p}^{T}(t) Z^{T} \bar{F}^{T} P_{2} \bar{F} Z \bar{x}_{p}(t) \\
& +2 e^{\beta t} \bar{x}_{p}^{T}(t) P_{2} \bar{D} Z \bar{x}_{m}(t)-2 e^{\beta t} \bar{x}_{p}^{T}(t) P_{2} \bar{D} Z \\
& \times\left[\int_{t-\tau_{2}}^{t} \xi_{m}(s) d s+\int_{t-\tau_{2}}^{t} \bar{E} Z \bar{x}_{m}(s) d \omega_{1}(s)\right] \\
& +e^{\beta\left(t+\tau_{2}\right)} \bar{x}_{m}^{T}(t) Z^{T} Q_{1} Z \bar{x}_{m}(t) \\
& -e^{\beta t} \bar{x}_{m}^{T}\left(t-\tau_{2}\right) Z^{T} Q_{1} Z \bar{x}_{m}\left(t-\tau_{2}\right) \\
& +e^{\beta\left(t+\tau_{1}\right)} \bar{x}_{p}^{T}(t) Z^{T} Q_{2} Z \bar{x}_{p}(t) \\
& -e^{\beta t} \bar{x}_{p}^{T}\left(t-\tau_{1}\right) Z^{T} Q_{2} Z \bar{x}_{p}\left(t-\tau_{1}\right) \\
& +e^{\beta t} \frac{e^{\beta \tau_{2}}-1}{\beta} \xi_{m}^{T}(t) Z^{T} R_{1} Z \xi_{m}(t) \\
& -e^{\beta t} \int_{t-\tau_{2}}^{t} \xi_{m}^{T}(s) Z^{T} R_{1} Z \xi_{m}(s) d s \\
& +e^{\beta t} \frac{e^{\beta \tau} 1}{\beta} \xi_{p}^{T}(t) Z^{T} R_{2} Z \xi_{p}(t) \\
& -e^{\beta t} \int_{t-\tau_{1}}^{t} \xi_{p}^{T}(s) Z^{T} R_{2} Z \xi_{p}(s) d s \\
& +\varepsilon_{1} e^{\beta t} \frac{e^{\beta \tau_{2}}-1}{\beta} \bar{x}_{m}^{T}(t) Z^{T} E^{T} E Z \bar{x}_{m}(t) \\
& -\varepsilon_{1} e^{\beta t} \int_{t-\tau_{2}}^{t} \bar{x}_{m}^{T}(s) Z^{T} E^{T} E Z \bar{x}_{m}(s) d s \\
& +\varepsilon_{2} e^{\beta t} \frac{e^{\beta \tau_{1}}-1}{\beta} \bar{x}_{p}^{T}(t) Z^{T} F^{T} F Z \bar{x}_{p}(t) \\
& -\varepsilon_{2} e^{\beta t} \int_{t-\tau_{1}}^{t} \bar{x}_{p}^{T}(s) Z^{T} F^{T} F Z \bar{x}_{p}(s) d s .
\end{aligned}
$$

From (3) and the definition of $f_{\tau_{1}}$ in (14), it can be easily seen that $f_{\tau_{1}} \leq K$. In terms of the fact that the positive definite matrices $Q_{i}, R_{i}$ and $S_{i}(i=1,2)$ are diagonal, we have from Lemma 2 that

$$
\begin{aligned}
2 \bar{x}_{m}^{T}(t) P_{1} \bar{B} f_{\tau_{1}} Z \bar{x}_{p}(t) \leq & \bar{x}_{m}^{T}(t) P_{1} \bar{B} S_{1}^{-1} \bar{B}^{T} P_{1} \bar{x}_{m}(t) \\
& +\bar{x}_{p}^{T}(t) Z^{T} f_{\tau_{1}}^{T} S_{1} f_{\tau_{1}} Z \bar{x}_{p}(t) \\
\leq & \bar{x}_{m}^{T}(t) P_{1} \bar{B} S_{1}^{-1} \bar{B}^{T} P_{1} \bar{x}_{m}(t) \\
& +\bar{x}_{p}^{T}(t) Z^{T} K S_{1} K Z \bar{x}_{p}(t) \\
-2 \bar{x}_{m}^{T}(t) P_{1} \bar{B} f_{\tau_{1}} Z \xi_{p}(s) \leq & \bar{x}_{m}^{T}(t) P_{1} \bar{B} f_{\tau_{1}} R_{2}^{-1} f_{\tau_{1}}^{T} \bar{B}^{T} P_{1} \bar{x}_{m}^{T}(t) \\
& +\xi_{p}^{T}(s) Z^{T} R_{2} Z \xi_{p}(s) \\
\leq & \bar{x}_{m}^{T}(t) P_{1} \bar{B} K R_{2}^{-1} K \bar{B}^{T} P_{1} \bar{x}_{m}^{T}(t) \\
& +\xi_{p}^{T}(s) Z^{T} R_{2} Z \xi_{p}(s) \\
2 \bar{x}_{p}^{T}(t) P_{2} \bar{D} Z \bar{x}_{m}(t) \leq & \bar{x}_{p}^{T}(t) P_{2} \bar{D} S_{2}^{-1} \bar{D}^{T} P_{2} \bar{x}_{p}(t) \\
& +\bar{x}_{m}^{T}(t) Z^{T} S_{2} Z \bar{x}_{m}(t) \\
-2 \bar{x}_{p}^{T}(t) P_{2} \bar{D} Z \xi_{m}(s) \leq & \bar{x}_{p}^{T}(t) P_{2} \bar{D} R_{1}^{-1} \bar{D}^{T} P_{2} \bar{x}_{p}^{T}(t) \\
& +\xi_{m}^{T}(s) Z^{T} R_{1} Z \xi_{m}(s) .
\end{aligned}
$$

Similarly, noticing that $Z \bar{E}=E, Z \bar{F}=F$ and $\varepsilon_{i}(i=1,2)$ are positive scalars, we obtain

$$
\begin{aligned}
\mathbb{E}\{ & \left.-2 \bar{x}_{m}^{T}(t) P_{1} \bar{B} f_{\tau_{1}} Z \int_{t-\tau_{1}}^{t} \bar{F} Z \bar{x}_{p}(s) d \omega_{2}(s)\right\} \\
= & \mathbb{E}\left\{-2 \bar{x}_{m}^{T}(t) P_{1} \bar{B} f_{\tau_{1}} \int_{t-\tau_{1}}^{t} F Z \bar{x}_{p}(s) d \omega_{2}(s)\right\} \\
\leq & \varepsilon_{2}^{-1} \mathbb{E}\left\{\bar{x}_{m}^{T}(t) P_{1} \bar{B} f_{\tau_{1}} f_{\tau_{1}}^{T} \bar{B}^{T} P_{1} \bar{x}_{m}^{T}(t)\right\} \\
& +\varepsilon_{2} \mathbb{E}\left|\int_{-\tau_{1}}^{t} F Z \bar{x}_{p}(s) d \omega_{2}(s)\right|^{2} \\
\leq & \varepsilon_{2}^{-1} \mathbb{E}\left\{\bar{x}_{m}^{T}(t) P_{1} \bar{B} K K \bar{B}^{T} P_{1} \bar{x}_{m}^{T}(t)\right\} \\
& +\varepsilon_{2} \mathbb{E} \int_{t-\tau_{1}}^{t} \bar{x}_{p}^{T}(s) Z^{T} F^{T} F Z \bar{x}_{p}^{T}(s) d s \\
\mathbb{E}\{ & \left.-2 \bar{x}_{p}^{T}(t) P_{2} \bar{D} Z \int_{t-\tau_{2}}^{t} \bar{E} Z \bar{x}_{m}(s) \omega_{1}(s) d s\right\} \\
= & \mathbb{E}\left\{-2 \bar{x}_{p}^{T}(t) P_{2} \bar{D} \int_{t-\tau_{2}}^{t} E Z \bar{x}_{m}(s) d \omega_{1}(s)\right\} \\
\leq & \varepsilon_{1}^{-1} \mathbb{E}\left\{\tau_{2} \bar{x}_{p}^{T}(t) P_{2} \bar{D} \bar{D}^{T} P_{2} \bar{x}_{p}^{T}(t)\right\} \\
& +\varepsilon_{1} \mathbb{E}\left|\int_{-\tau_{2}}^{t} E Z \bar{x}_{m}(s) d \omega_{1}(s)\right|^{2} \\
\leq & \varepsilon_{1}^{-1} \mathbb{E}\left\{\tau_{2} \bar{x}_{p}^{T}(t) P_{2} \bar{D} \bar{D}^{T} P_{2} \bar{x}_{p}^{T}(t)\right\} \\
& +\varepsilon_{1} \mathbb{E} \int_{t}^{t} \bar{x}_{m}^{T}(s) Z^{T} E^{T} E Z \bar{x}_{m}(s) d s .
\end{aligned}
$$

Noting the sector condition (4), we substitute (18)-(20) into (17), take mathematical expectation on both sides and obtain

$$
\begin{aligned}
& \mathbb{E}\{\mathcal{L} V(t)\} \\
& \leq e^{\beta t} \mathbb{E}\left\{\overline { x } _ { m } ^ { T } ( t ) \left(\bar{A}^{T} P_{1}+P_{1} \bar{A}+P_{1} \bar{B} S_{1}^{-1} \bar{B}^{T} P_{1}\right.\right. \\
& +\tau_{1} P_{1} \bar{B} K R_{2}^{-1} K \bar{B}^{T} P_{1} \\
& +\varepsilon_{2}^{-1} P_{1} \bar{B} K^{2} \bar{B}^{T} P_{1}+Z^{T} \bar{E}^{T} P_{1} \bar{E} Z \\
& +e^{\beta \tau_{2}} Z^{T} Q_{1} Z+Z^{T} S_{2} Z \\
& \left.+\varepsilon_{1} \frac{e^{\beta \tau_{2}}-1}{\beta} Z^{T} E^{T} E Z+\beta P_{1}\right) \bar{x}_{m}^{T}(t) \\
& +\bar{x}_{p}^{T}(t)\left(\bar{C}^{T} P_{2}+P_{2} \bar{C}+P_{2} \bar{D} S_{2}^{-1} \bar{D}^{T} P_{2}\right. \\
& +\tau_{2} P_{2} \bar{D} R_{1}^{-1} \bar{D}^{T} P_{2}+\varepsilon_{1}^{-1} P_{2} \bar{D} \bar{D}^{T} P_{2} \\
& +Z^{T} \bar{F}^{T} P_{2} \bar{F} Z+e^{\beta \tau_{1}} Z^{T} Q_{2} Z \\
& +Z^{T} K S_{1} K Z+\varepsilon_{2} \frac{e^{\beta \tau_{1}}-1}{\beta} Z^{T} F^{T} F Z \\
& \left.+\beta P_{2}\right) \bar{x}_{p}(t)-2 g^{T}\left(Z \bar{x}_{p \tau_{1}}\right) \\
& \times\left(g\left(Z \bar{x}_{p \tau_{1}}\right)-K Z \bar{x}_{p \tau_{1}}\right)-\bar{x}_{m \tau_{2}}^{T} Z^{T} Q_{1} Z \bar{x}_{m \tau_{2}} \\
& -\bar{x}_{p \tau_{1}}^{T} Z^{T} Q_{2} Z \bar{x}_{p \tau_{1}}+\frac{e^{\beta \tau_{2}}-1}{\beta} \\
& \times\left[\bar{A} \bar{x}_{m}(t)+\bar{B} g\left(Z \bar{x}_{p \tau_{1}}\right)\right]^{T} Z^{T} R_{1} Z
\end{aligned}
$$




$$
\begin{aligned}
& \times\left[\bar{A} \bar{x}_{m}(t)+\bar{B} g\left(Z \bar{x}_{p \tau_{1}}\right)\right]+\frac{e^{\beta \tau_{1}}-1}{\beta} \\
& \times\left[\bar{C} \bar{x}_{p}(t)+\bar{D} Z \bar{x}_{m \tau_{2}}\right]^{T} Z^{T} R_{2} Z \\
&\left.\left.\times\left[\bar{C} \bar{x}_{p}(t)+\bar{D} Z \bar{x}_{m \tau_{2}}\right)\right]\right\} . \\
& \leq e^{\beta t} \mathbb{E}\left\{\eta^{T}(t) \Gamma \eta(t)\right\}
\end{aligned}
$$

where we have (22), shown at the bottom of page, with

$$
\begin{aligned}
\Gamma_{1}= & \bar{A}^{T} P_{1}+P_{1} \bar{A}+P_{1} \bar{B} S_{1}^{-1} \bar{B}^{T} P_{1}+\tau_{1} P_{1} \bar{B} K R_{2}^{-1} K \bar{B}^{T} P_{1} \\
& +\varepsilon_{2}^{-1} P_{1} \bar{B} K^{2} \bar{B}^{T} P_{1}+Z^{T} \bar{E}^{T} P_{1} \bar{E} Z \\
& +\frac{e^{\beta \tau_{2}}-1}{\beta} \bar{A}^{T} Z^{T} R_{1} Z \bar{A}+e^{\beta \tau_{2}} Z^{T} Q_{1} Z+Z^{T} S_{2} Z \\
& +\varepsilon_{1} \frac{e^{\beta \tau_{2}}-1}{\beta} Z^{T} E^{T} E Z+\beta P_{1} \\
\Gamma_{2}= & \bar{C}^{T} P_{2}+P_{2} \bar{C}+P_{2} \bar{D} S_{2}^{-1} \bar{D}^{T} P_{2}+\tau_{2} P_{2} \bar{D} R_{1}^{-1} \bar{D}^{T} P_{2} \\
& +\varepsilon_{1} P_{1}^{-1} \bar{D} \bar{D}^{T} P_{2}+Z^{T} \bar{F}^{T} P_{2} \bar{F} Z \\
& +\frac{e^{\beta \tau_{1}}-1}{\beta} \bar{C}^{T} Z^{T} R_{2} Z \bar{C}+e^{\beta \tau_{1}} Z^{T} Q_{2} Z+Z^{T} K S_{1} K Z \\
& +\varepsilon_{2} \frac{e^{\beta \tau_{1}}-1}{\beta} Z^{T} F^{T} F Z+\beta P_{2} .
\end{aligned}
$$

By Schur complement, we can conclude from (10) that $\Gamma<0$ and therefore $\mathbb{E}\{\mathcal{L} V(t)\}<0$. We are now ready to prove the mean square exponential stability of system (13). Define

$$
\begin{aligned}
\lambda_{p} & =\min \left\{\lambda_{\min }\left(P_{1}\right), \lambda_{\min }\left(P_{2}\right)\right\} \\
\|P\| & =\max \left\{\left\|P_{1}\right\|,\left\|P_{2}\right\|\right\} \\
\|Q\| & =\max \left\{\left\|Q_{1}\right\|,\left\|Q_{2}\right\|\right\},\|R\|=\max \left\{\left\|R_{1}\right\|,\left\|R_{2}\right\|\right\} \\
\varepsilon & =\max \left\{\varepsilon_{1}, \varepsilon_{2}\right\} .
\end{aligned}
$$

From the definitions of $\xi_{m}(t), \xi_{p}(t), \bar{x}(t), \rho(t)$ and (15), it follows that there exists a positive scalar $\delta$ such that

$$
\mathbb{E} V(t) \geq e^{\beta t} \lambda_{p} \mathbb{E}|\bar{x}(t)|^{2}
$$

and

$$
\begin{aligned}
\mathbb{E} V(0) \leq\{ & \|P\|+\tau e^{\beta \tau}\|Q\| \\
& +\tau^{2} e^{\beta \tau}\left[2 \| R \| \left(\|\bar{A}\|^{2}\right.\right. \\
& \left.+\|K\|^{2}\|\bar{B}\|^{2}+\|\bar{C}\|^{2}+\|\bar{D}\|^{2}\right) \\
& \left.\left.+\varepsilon\|E\|^{2}+\varepsilon\|F\|^{2}\right]\right\} \sup _{-2 \tau \leq t \leq 0}|\rho(t)|^{2} \\
\leq & \sup _{-2 \tau \leq t \leq 0} \mathbb{E}|\rho(t)|^{2} .
\end{aligned}
$$

By Itô's formula [21], we obtain that

$$
\mathbb{E} V(t)=\mathbb{E} V(0)+\mathbb{E} \int_{0}^{t} \mathcal{L} V(s) d s \leq \delta \sup _{-2 \tau \leq t \leq 0} \mathbb{E}|\rho(t)|^{2} .
$$

It follows from (23), (24) and (25) that

$$
e^{\beta t} \lambda_{p} \mathbb{E}|\bar{x}(t, \rho)|^{2} \leq \delta \sup _{-2 \tau \leq t \leq 0} \mathbb{E}|\rho(t)|^{2}
$$

or, equivalently,

$$
\lim _{t \rightarrow \infty} \sup \frac{1}{t} \log \left(\mathbb{E}|\bar{x}(t, \rho)|^{2}\right) \leq-\beta,
$$

which indicates that the trivial solution of (13) is exponentially mean square stable and the exponential decay rate is $\beta$. The proof is now complete.

Remark 3: In Theorem 1, the lower bound $\beta$ for the exponential decay rate is given in the criterion. Such a lower bound behaves as a flexible performance parameter adjustable according to different practical applications. Note that the decay rate lower bound characterization approach has been exploited in [27] for a class of stochastic neural networks with time delay.

Remark 4: In Theorem 1, given filter parameters and decay rate lower bound $\beta,(10)$ is a linear matrix inequality whose feasibility can be readily checked by using the Matlab LMI toolbox [10].

\section{B. Filter Synthesis}

The following theorem shows that the desired filter parameters can be determined by solving an LMI.

Theorem 2: Consider system (6). For a prescribed constant $\beta>0$, if there exist matrices $X_{i}>0, \mathcal{Y}_{i}>0$, diagonal matrices $Q_{i}>0$, $R_{i}>0, S_{i}>0$ and scalars $\varepsilon_{i}>0(i=1,2)$ such that the linear matrix inequality (26), shown at the bottom of the next page, holds, where

$$
\begin{aligned}
& \Pi_{11}:=-A_{1}^{T} \mathcal{Y}_{1}-\mathcal{Y}_{1} A_{1}+\beta \mathcal{Y}_{1} \\
& \Pi_{12}:=-A_{1}^{T} X_{1}-\mathcal{Y}_{1} A_{1}+C_{1}^{T} \tilde{B}^{T}+\tilde{A}^{T}+\beta \mathcal{Y}_{1} \\
& \Pi_{22}:=-A_{1}^{T} X_{1}-X_{1} A_{1}+\tilde{B} C_{1}+C_{1}^{T} \tilde{B}^{T}+\beta X_{1} \\
& \Pi_{33}:=-A_{2}^{T} \mathcal{Y}_{2}-\mathcal{Y}_{2} A_{2}+\beta \mathcal{Y}_{2} \\
& \Pi_{34}:=-A_{2}^{T} X_{2}-\mathcal{Y}_{2} A_{2}+C_{2}^{T} \tilde{D}^{T}+\tilde{C}^{T}+\beta \mathcal{Y}_{2} \\
& \Pi_{44}:=-A_{2}^{T} X_{2}-X_{2} A_{2}+\tilde{D} C_{2}+C_{2}^{T} \tilde{D}^{T}+\beta X_{2} \\
& \Phi_{1}:=\left[\begin{array}{lllll}
\mathcal{Y}_{1} B & \tau_{1} \mathcal{Y}_{1} B K & \mathcal{Y}_{1} B K & \varepsilon_{1} E^{T} & Q_{1} S_{2}
\end{array}\right] \\
& \Phi_{5}:=\left[\begin{array}{lll}
E^{T} \mathcal{Y}_{1} & E^{T} X_{1}
\end{array}\right] \\
& \Phi_{2}:=\left[\begin{array}{llllll}
X_{1} B & \tau_{1} X_{1} B K & X_{1} B K & \varepsilon_{1} E^{T} & Q_{1} & S_{2}
\end{array}\right] \\
& \Phi_{6}:=\left[\begin{array}{lll}
E^{T} \mathcal{Y}_{1} & E^{T} X_{1}
\end{array}\right]
\end{aligned}
$$

$$
\begin{aligned}
& \eta(t)=\left[\bar{x}_{m}^{T}(t) \bar{x}_{p}^{T}(t) Z \bar{x}_{m \tau_{2}}^{T} Z \bar{x}_{p \tau_{1}}^{T} g\left(Z \bar{x}_{p \tau_{1}}\right)\right]^{T} \\
& \Gamma=\left[\begin{array}{ccccc}
\Gamma_{1} & 0 & 0 & 0 & \frac{e^{\beta \tau_{2}}-1}{\beta} \bar{A}^{T} Z^{T} R_{1} Z \bar{B} \\
* & \Gamma_{2} & \frac{e^{\beta \tau}-1}{\beta} \bar{C}^{T} Z^{T} R_{2} Z \bar{D} & 0 & 0 \\
* & * & -Q_{1}+\frac{e^{\beta \tau} \tau_{1}-1}{\beta} \bar{D}^{T} Z^{T} R_{2} Z \bar{D} & 0 & 0 \\
* & * & * & -Q_{2} & K \\
* & * & * & * & -2 I+\frac{e^{\beta \tau_{2}-1}}{\beta} \bar{B}^{T} Z^{T} R_{1} Z \bar{B}
\end{array}\right]
\end{aligned}
$$




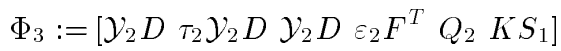

$$
\begin{aligned}
& \Phi_{7}:=\left[\begin{array}{lll}
F^{T} \mathcal{Y}_{2} & F^{T} X_{2}
\end{array}\right]
\end{aligned}
$$

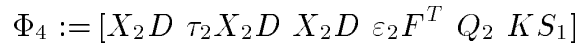

$$
\begin{aligned}
& \Phi_{8}:=\left[\begin{array}{lll}
F^{T} \mathcal{Y}_{2} & F^{T} X_{2}
\end{array}\right] \\
& \Lambda_{1}:=\operatorname{diag}\left\{S_{1}, \tau_{1} R_{2}, \varepsilon_{2} I, \frac{\varepsilon_{1} \beta}{e^{\beta \tau_{2}}-1} I, e^{-\beta \tau_{2}} Q_{1}, S_{2}\right\} \\
& \Lambda_{3}:=\left[\begin{array}{ll}
\mathcal{Y}_{1} & \mathcal{Y}_{1} \\
\mathcal{Y}_{1} & X_{1}
\end{array}\right] \\
& \Lambda_{2}:=\operatorname{diag}\left\{S_{2}, \tau_{2} R_{1}, \varepsilon_{1} I, \frac{\varepsilon_{2} \beta}{e^{\beta \tau_{1}}-1} I, e^{-\beta \tau_{1}} Q_{2}, S_{1}\right\} \\
& \Lambda_{4}:=\left[\begin{array}{ll}
\mathcal{Y}_{2} & \mathcal{Y}_{2} \\
\mathcal{Y}_{2} & X_{2}
\end{array}\right]
\end{aligned}
$$

then system (6) is exponentially mean square stable. In this case, the parameters of the desired filter $\left(\Sigma_{f}\right)$ are given as follows:

$$
\begin{aligned}
& \hat{A}:=\left(\mathcal{Y}_{1}-X_{1}\right)^{-1} \tilde{A}, \hat{B}:=\left(\mathcal{Y}_{1}-X_{1}\right)^{-1} \tilde{B} \\
& \hat{C}:=\left(\mathcal{Y}_{2}-X_{2}\right)^{-1} \tilde{C}, \hat{D}:=\left(\mathcal{Y}_{2}-X_{2}\right)^{-1} \tilde{D} .
\end{aligned}
$$

Proof: Define

$$
P_{i}=\left[\begin{array}{cc}
X_{i} & \mathcal{Y}_{i}-X_{i} \\
\mathcal{Y}_{i}-X_{i} & X_{i}-\mathcal{Y}_{i}
\end{array}\right]>0, \quad \Upsilon_{i}=\left[\begin{array}{cc}
Y_{i} & I \\
Y_{i} & 0
\end{array}\right], \quad i=1,2,
$$

where $Y_{i}=\mathcal{Y}_{i}^{-1}>0$.
Pre- and post-multiplying the LMI in (26) by

$\operatorname{diag}\left\{Y_{1}, I, Y_{2}, I, I, I, I, I, I, \operatorname{diag}_{6}\{I\}, \operatorname{diag}_{6}\{I\}\right.$,

$$
\left.\operatorname{diag}\left\{Y_{1}, I\right\}, \operatorname{diag}\left\{Y_{2}, I\right\}\right\}
$$

we have (30), shown at the bottom of the page, where

$$
\begin{aligned}
& \bar{\Pi}_{11}:=-Y_{1} A_{1}^{T}-A_{1} Y_{1}+\beta Y_{1} \\
& \bar{\Pi}_{12}:=-Y_{1} A_{1}^{T} X_{1}-A_{1}+Y_{1} C_{1}^{T} \tilde{B}^{T}+Y_{1} \tilde{A}^{T}+\beta I \\
& \bar{\Pi}_{33}:=-Y_{2} A_{2}^{T}-A_{2} Y_{2}+\beta Y_{2} \\
& \bar{\Pi}_{34}:=-Y_{2} A_{2}^{T} X_{2}-A_{2}+Y_{2} C_{2}^{T} \tilde{D}^{T}+Y_{2} \tilde{C}^{T}+\beta I \\
& \bar{\Phi}_{1}:=\left[\begin{array}{llllll}
B & \tau_{1} B K & B K & \varepsilon_{1} Y_{1} E^{T} & Y_{1} Q_{1} & Y_{1} S_{2}
\end{array}\right] \\
& \bar{\Phi}_{5}:=\left[Y_{1} E^{T} Y_{1} E^{T} X_{1}\right]
\end{aligned}
$$

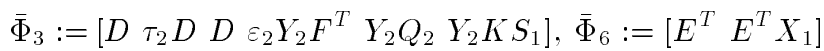

$$
\begin{aligned}
& \bar{\Phi}_{7}:=\left[Y_{2} F^{T} Y_{2} F^{T} X_{2}\right], \bar{\Phi}_{8}:=\left[\begin{array}{ll}
F^{T} & F^{T} X_{2}
\end{array}\right] \\
& \operatorname{bar}_{3}:=\left[\begin{array}{cc}
Y_{1} & I \\
I & X_{1}
\end{array}\right], \bar{\Lambda}_{4}:=\left[\begin{array}{cc}
Y_{2} & I \\
I & X_{2}
\end{array}\right] \text {. }
\end{aligned}
$$

$$
\left[\begin{array}{ccccccccccccc}
\Pi_{11} & \Pi_{12} & 0 & 0 & 0 & 0 & 0 & -A_{1}^{T} R_{1} & 0 & \Phi_{1} & 0 & \Phi_{5} & 0 \\
* & \Pi_{22} & 0 & 0 & 0 & 0 & 0 & -A_{1}^{T} R_{1} & 0 & \Phi_{2} & 0 & \Phi_{6} & 0 \\
* & * & \Pi_{33} & \Pi_{34} & 0 & 0 & 0 & 0 & -A_{2}^{T} R_{2} & 0 & \Phi_{3} & 0 & \Phi_{7} \\
* & * & * & \Pi_{44} & 0 & 0 & 0 & 0 & -A_{2}^{T} R_{2} & 0 & \Phi_{4} & 0 & \Phi_{8} \\
* & * & * & * & -Q_{1} & 0 & 0 & 0 & D^{T} R_{2} & 0 & 0 & 0 & 0 \\
* & * & * & * & * & -Q_{2} & K & 0 & 0 & 0 & 0 & 0 & 0 \\
* & * & * & * & * & * & -2 I & B^{T} R_{1} & 0 & 0 & 0 & 0 & 0 \\
* & * & * & * & * & * & * & -\frac{\beta}{e^{\beta} \tau_{2}-1} R_{1} & 0 & 0 & 0 & 0 & 0 \\
* & * & * & * & * & * & * & * & -\frac{\beta}{e^{\beta \tau} 1-1} R_{2} & 0 & 0 & 0 & 0 \\
* & * & * & * & * & * & * & * & * & -\Lambda_{1} & 0 & 0 & 0 \\
* & * & * & * & * & * & * & * & * & * & -\Lambda_{2} & 0 & 0 \\
* & * & * & * & * & * & * & * & * & * & * & -\Lambda_{3} & 0 \\
* & * & * & * & * & * & * & * & * & * & * & * & -\Lambda_{4}
\end{array}\right]
$$

$$
\left[\begin{array}{ccccccccccccc}
\bar{\Pi}_{11} & \bar{\Pi}_{12} & 0 & 0 & 0 & 0 & 0 & -Y_{1} A_{1}^{T} R_{1} & 0 & \bar{\Phi}_{1} & 0 & \bar{\Phi}_{5} & 0 \\
* & \Pi_{22} & 0 & 0 & 0 & 0 & 0 & -A_{1}^{T} R_{1} & 0 & \Phi_{2} & 0 & \bar{\Phi}_{6} & 0 \\
* & * & \bar{\Pi}_{33} & \bar{\Pi}_{34} & 0 & 0 & 0 & 0 & -Y_{2} A_{2}^{T} R_{2} & 0 & \bar{\Phi}_{3} & 0 & \bar{\Phi}_{7} \\
* & * & * & \Pi_{44} & 0 & 0 & 0 & 0 & -A_{2}^{T} R_{2} & 0 & \Phi_{4} & 0 & \bar{\Phi}_{8} \\
* & * & * & * & -Q_{1} & 0 & 0 & 0 & D^{T} R_{2} & 0 & 0 & 0 & 0 \\
* & * & * & * & * & -Q_{2} & K & 0 & 0 & 0 & 0 & 0 & 0 \\
* & * & * & * & * & * & -2 I & B^{T} R_{1} & 0 & 0 & 0 & 0 & 0 \\
* & * & * & * & * & * & * & -\frac{\beta}{e^{\beta} \tau_{2}-1} R_{1} & 0 & 0 & 0 & 0 & 0 \\
* & * & * & * & * & * & * & * & -\frac{\beta}{e^{\beta \tau} 1-1} R_{2} & 0 & 0 & 0 & 0 \\
* & * & * & * & * & * & * & * & * & -\Lambda_{1} & 0 & 0 & 0 \\
* & * & * & * & * & * & * & * & * & * & -\Lambda_{2} & 0 & 0 \\
* & * & * & * & * & * & * & * & * & * & * & -\bar{\Lambda}_{3} & 0 \\
* & * & * & * & * & * & * & * & * & * & * & * & -\bar{\Lambda}_{4}
\end{array}\right]
$$


It can be seen from the definitions of $P_{1}, P_{2}, \Upsilon_{1}$ and $\Upsilon_{2}$ that the LMI in (30) is equivalent to (31), shown at the bottom of the page, where

$$
\begin{aligned}
& \Psi_{1}:=\Upsilon_{1}^{T} \bar{A}^{T} Z^{T} R_{1}, \quad \Psi_{2}:=\bar{B}^{T} Z^{T} R_{1} \\
& \Psi_{3}:=\Upsilon_{2}^{T} \bar{C}^{T} Z^{T} R_{2}, \quad \Psi_{4}:=\bar{D}^{T} Z^{T} R_{2} \\
& \Psi_{5}:=\Upsilon_{1}^{T} Z^{T} \bar{E}^{T} P_{1} \Upsilon_{1}, \quad \Psi_{6}:=\Upsilon_{2}^{T} Z^{T} \bar{F}^{T} P_{2} \Upsilon_{2} \\
& \Delta_{1}:=\Upsilon_{1}^{T} P_{1} \Upsilon_{1}, \quad \Delta_{2}:=\Upsilon_{2}^{T} P_{2} \Upsilon_{2} .
\end{aligned}
$$

Finally, pre- and post-multiplying (31) by $\operatorname{diag}\left\{\Upsilon_{1}^{-T}, \Upsilon_{2}^{-T}, I, I, I, I, I, \operatorname{diag}_{5}\{I\}, \operatorname{diag}_{5}\{I\}, \Upsilon_{1}^{-T}, \Upsilon_{2}^{-T}\right\}$ and its transpose, we can obtain from Theorem 1 and Schur complement that system (6) is exponentially mean square stable with the given filter parameters in (28).

\section{AN Illustrative EXAMPLE}

In this section, a simulation example is presented to illustrate the usefulness and flexibility of the filter design method developed in this paper.

The dynamics of repressilator has been theoretically predicted and experimentally investigated in Escherichia coli [19]. The repressilator is a cyclic negative-feedback loop comprising three repressor genes (lacl, tetR and $\mathrm{cl}$ ) and their promoters. The kinetics of the system are described as follows:

$$
\left\{\begin{array}{l}
\dot{x}_{m_{\text {lacl }}}=-x_{m_{\text {lacl }}}+\frac{a}{1+x_{p_{\mathrm{cl}}}^{n}} \\
\dot{x}_{p_{\text {lacl }}}=-b\left(x_{p_{\text {lacl }}}-x_{m_{\text {lacl }}}\right) \\
\dot{x}_{m_{\mathrm{tetR}}}=-x_{m_{\mathrm{tetR}}}+\frac{a}{1+x_{p_{\mathrm{lacl}}}^{n}} \\
\dot{x}_{p_{\mathrm{tetR}}}=-b\left(x_{p_{\mathrm{tetR}}}-x_{m_{\mathrm{tetR}}}\right) \\
\dot{x}_{m_{\mathrm{cl}}}=-x_{m_{\mathrm{cl}}}+\frac{a}{1+x_{p_{\mathrm{tetR}}}^{n}} \\
\dot{x}_{p_{\mathrm{cl}}}=-b\left(x_{p_{\mathrm{cl}}}-x_{m_{\mathrm{cl}}}\right),
\end{array}\right.
$$

where $x_{m_{i}}$ and $x_{p_{i}}(i=1 \mathrm{acl}$, tetR and $\mathrm{cl})$ are the concentrations of the three mRNA and repressor protein, $b>0$ denotes the ratio of the protein decay rate to mRNA decay rate, and $a$ is the feedback regulation coefficient. Taking into account the time-delay and stochastic disturbance, we consider the following compact matrix form nonlinear genetic regulatory network model (1):

$$
\left\{\begin{array}{l}
d x_{m}(t)=\left[-A_{1} x_{m}(t)+B g\left(x_{p}\left(t-\tau_{1}\right)\right)\right] d t+E x_{m}(t) d \omega_{1}(t) \\
d x_{p}(t)=\left[-A_{2} x_{p}(t)+D x_{m}\left(t-\tau_{2}\right)\right] d t+F x_{p}(t) d \omega_{2}(t) \\
y_{m}(t)=C_{1} x_{m}(t) \\
y_{p}(t)=C_{2} x_{p}(t),
\end{array}\right.
$$

with transcriptional time delay $\tau_{1}=0.3, \tau_{2}=0.7$, exponential decay rate lower bound $\beta=1.0$ as well as the following data:

$$
\begin{aligned}
A_{1} & =\operatorname{diag}\{3,3,3\}, C_{1}=\operatorname{diag}\{0.72,0.1,1.3\} \\
B & =\left[\begin{array}{ccc}
0 & 0 & -1.2 \\
-0.02 & 0 & 0 \\
0 & -0.2 & 0
\end{array}\right] \\
A_{2} & =\operatorname{diag}\{3.5,3.5,3.5\}, C_{2}=\operatorname{diag}\{1.2,3.1,0.03\} \\
D & =\operatorname{diag}\{1.21,2.12,0.22\} \\
E & =\operatorname{diag}\{0.53,0.41,0.42\}, F=\operatorname{diag}\{0.51,0.41,0.42\} \\
K & =\operatorname{diag}\{0.6,0.6,0.6\},
\end{aligned}
$$

where the sector-like nonlinearities are taken as

$$
g_{i}\left(x_{p_{i}}\right)=x_{p_{i}}^{2} /\left(1+x_{p_{i}}^{2}\right), \quad i=1,2, \ldots, n .
$$

It is easy to check that the maximal value of the derivative of $g_{i}\left(x_{p_{i}}\right)$ is less than $k_{i}=0.6$.

By using the LMI toolbox [10], we solve (26) and obtain

$$
\begin{aligned}
X_{1} & =\operatorname{diag}\{89.2144,899.4217,436.4357\} \\
X_{2} & =\operatorname{diag}\{3.5167,28.2677,492.5005\} \\
Y_{1} & =\operatorname{diag}\{29.1878,419.5382,186.9026\} \\
Y_{2} & =\operatorname{diag}\{1.1156,8.5315,231.5299\} \\
Q_{1} & =\operatorname{diag}\{1.1973,109.4880,47.2546\} \\
Q_{2} & =\operatorname{diag}\{0.2548,2.3778,97.5095\} \\
R_{1} & =\operatorname{diag}\{0.7410,55.6729,12.1232\} \\
R_{2} & =\operatorname{diag}\{0.0717,1.5388,62.9470\} \\
S_{1} & =\operatorname{diag}\{0.6005,16.8998,593.8720\} \\
S_{2} & =\operatorname{diag}\{6.8134,256.8588,103.3940\} \\
\varepsilon_{1} & =22.2904, \varepsilon_{2}=13.0905 .
\end{aligned}
$$

According to Theorem 2, the filter parameters can be calculated as follows:

$$
\begin{aligned}
& \hat{A}=\operatorname{diag}\{-8.0781,-1.8193,-2.9005\} \\
& \hat{B}=\operatorname{diag}\{8.8710,-7.9237,0.6790\} \\
& \hat{C}=\operatorname{diag}\{-159.4923,-22.3503,-2.7487\} \\
& \hat{D}=\operatorname{diag}\{131.5371,6.8160,6.7016\} .
\end{aligned}
$$

$\left[\begin{array}{ccccccccccc}\Upsilon_{1}^{T} \Omega_{1} \Upsilon_{1} & 0 & 0 & 0 & 0 & \Psi_{1} & 0 & \Upsilon_{1}^{T} \mathcal{U}_{1} & 0 & \Psi_{5} & 0 \\ * & \Upsilon_{2}^{T} \Omega_{2} \Upsilon_{2} & 0 & 0 & 0 & 0 & \Psi_{3} & 0 & \Upsilon_{2}^{T} \mathcal{U}_{2} & 0 & \Psi_{6} \\ * & * & -Q_{1} & 0 & 0 & 0 & \Psi_{4} & 0 & 0 & 0 & 0 \\ * & * & * & -Q_{2} & K & 0 & 0 & 0 & 0 & 0 & 0 \\ * & * & * & * & -2 I & \Psi_{2} & 0 & 0 & 0 & 0 & 0 \\ * & * & * & * & * & -\frac{\beta}{e^{\beta} \tau_{2}-1} R_{1} & 0 & 0 & 0 & 0 & 0 \\ * & * & * & * & * & * & -\frac{\beta}{e^{\beta} \tau_{2}-1} R_{2} & 0 & 0 & 0 & 0 \\ * & * & * & * & * & * & * & -\mathcal{V}_{1} & 0 & 0 & 0 \\ * & * & * & * & * & * & * & * & -\mathcal{V}_{2} & 0 & 0 \\ * & * & * & * & * & * & * & * & * & -\Delta_{1} & 0 \\ * & * & * & * & * & * & * & * & * & * & -\Delta_{2}\end{array}\right]$




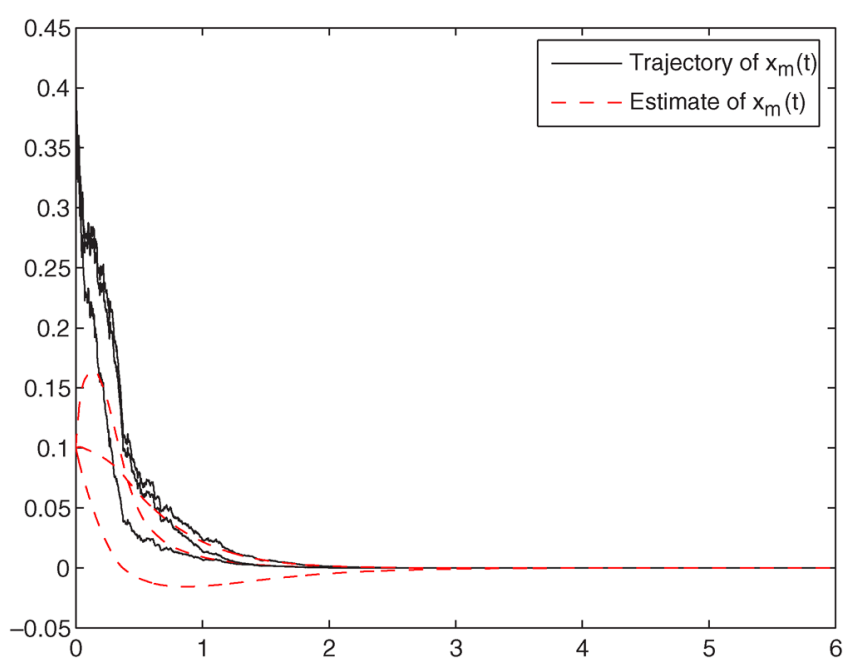

Fig. 1. Trajectory and estimate of $\boldsymbol{x}_{m}(\boldsymbol{t})$.

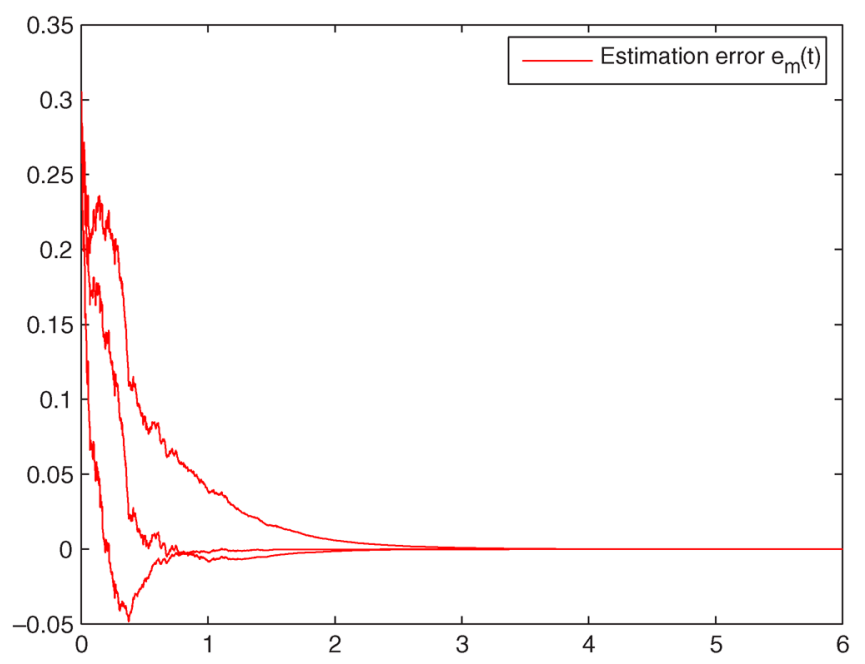

Fig. 2. Estimation error of $\boldsymbol{e}_{m}(\boldsymbol{t})$.

Figs. 1-4 give the simulation results for the performance of the designed filter, Fig. 5 gives the simulation for the actual decay rate of augmented systems state $\bar{x}(t)$ via $-\sup (1 / t) \log \left(\mathbb{E}|\bar{x}(t, \rho)|^{2}\right)$ and the prescribed decay rate lower bound $\beta$, where the initial condition $\rho(t)=$

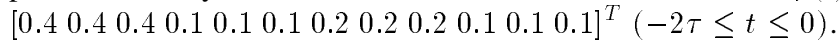

It can be seen from Fig. 5 that, as the time is greater than 1.2, the actual decay rate of the augmented system state $\bar{x}(t)$ becomes greater than its prescribed lower bound $\beta=1$. This clearly illustrates that the expected performance of exponential mean square stability is well achieved with the given decay rate lower bound.

\section{CONCLUSION}

In this paper, we have investigated the filtering problem on a class of stochastic time-delay genetic regulatory networks with sector-like nonlinearity, where both the translation delay and feedback regulation delay have been taken into account. By using Itô's differential formula and Lyapunov stability theory, we have proposed a linear matrix inequality method to derive sufficient conditions under which the desired filters exist. We have also characterized the expression of the filter parameters and the decay rate lower bound $\beta>0$, and employed a simulation example to illustrate the effectiveness of the proposed results. It should be pointed out that, we can extend the main results in this paper

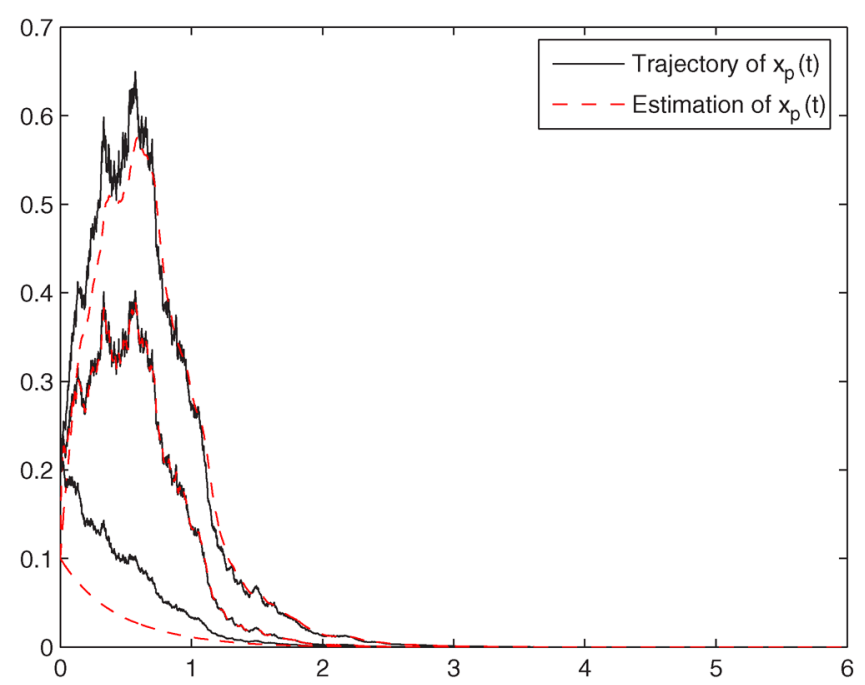

Fig. 3. Trajectory and estimate of $\boldsymbol{x}_{p}(\boldsymbol{t})$.

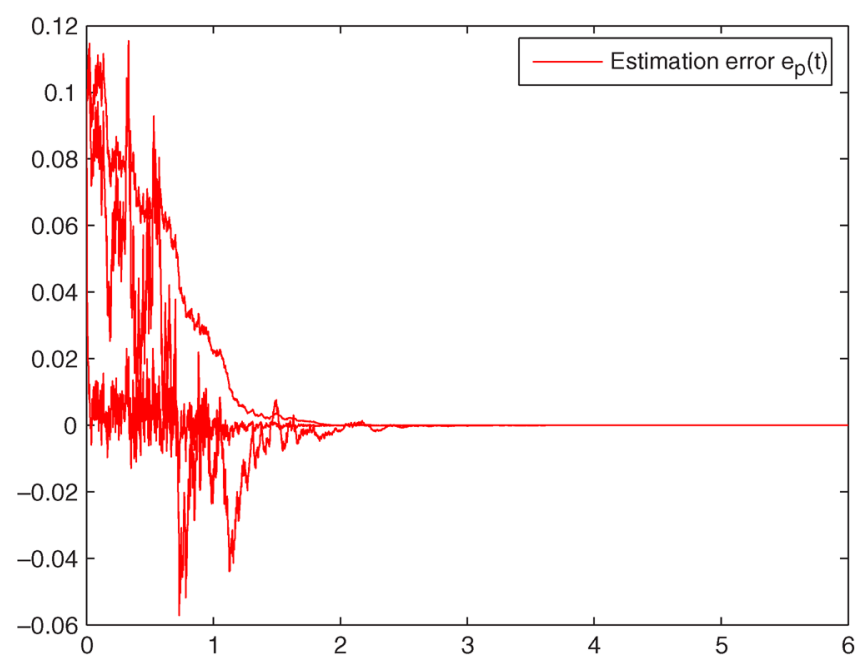

Fig. 4. Estimation error $\boldsymbol{e}_{p}(\boldsymbol{t})$.

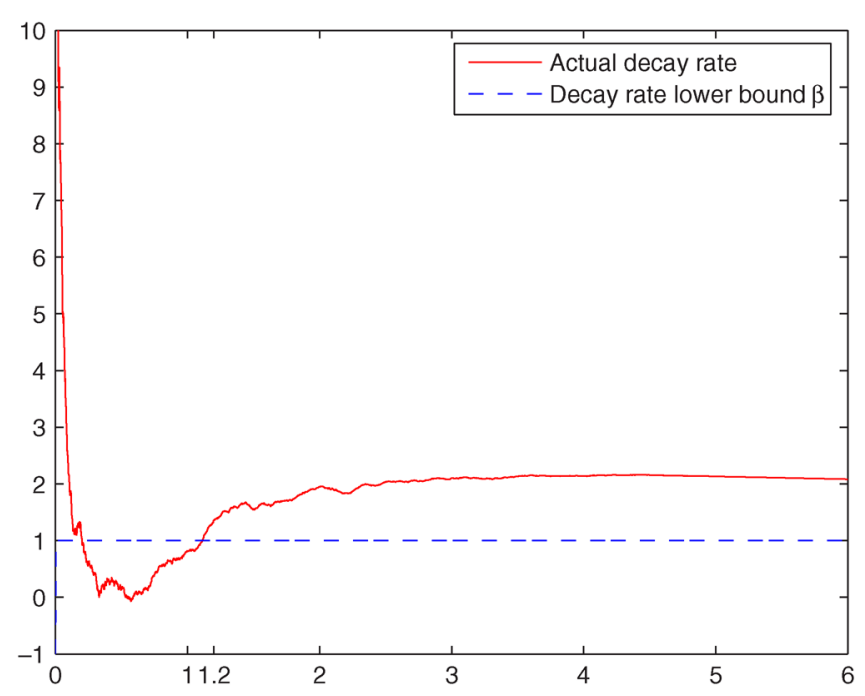

Fig. 5. Actual decay rate of $\overline{\boldsymbol{x}}(\boldsymbol{t})$ and decay rate lower bound $\boldsymbol{\beta}$.

to more complex and realistic systems, such as systems with polytopic or norm-bounded uncertainties. 


\section{REFERENCES}

[1] M. Barrio, K. Burrage, A. Leier, and T. Tian, "Oscillatory regulation of Hes1: Discrete stochastic delay modelling and simulation," PLOS Computat. Biol., vol. 2, no. 9, pp. 1017-1030, Sep. 2006.

[2] S. Boyd, L. EI Ghaoui, E. Feron, and V. Balakrishnan, Linear Matrix Inequalities in Systems and Control Theory. Philadelphia, PA: SIAM, 1994.

[3] B.-S. Chen and Y. Wang, "On the attenuation and amplification of molecular noise in genetic regulatory networks," BMC Bioinformatics, vol. 7, no. 52, pp. 1-14, Feb. 2006.

[4] T. Chen, H. He, and G. Church, "Modeling gene expression with differential equations," in Proc. Pacific Symp. Biocomput., 1999, vol. 4, pp. 29-40.

[5] L. Chen and K. Aihara, "Stability of genetic regulatory networks with time delay," IEEE Trans. Circuits Syst. I, vol. 49, no. 5, pp. 602-608, May 2002.

[6] D. Cook, A. Gerber, and S. Tapscott, "Modeling stochastic gene expression: Implications for haploinsufficiency," Proc. Nat. Acad. Sci., vol. 95, pp. 15641-15646, 1998.

[7] J. Cao and F. Ren, "Exponential stability of discrete-time genetic regulatory networks with delays," IEEE Trans. Neural Networks, vol. 19, no. 3, pp. 520-523, Mar. 2008.

[8] P. D'haeseleer, X. Wen, S. Fuhrman, and R. Somogyi, "Linear modeling of mRNA expression levels during CNS development and injury," in Proc. Pacific Symp. Biocomput., 1999, vol. 4, pp. 41-52.

[9] M. B. Elowitz and S. Leibler, "A synthetic oscillatory network of transcriptional regulators," Nature, vol. 403, pp. 335-338, 2000.

[10] P. Gahinet, A. Nemirovsky, A. J. Laub, and M. Chilali, LMI Control Toolbox: For Use With Matlab.. New York: The Math Works, Inc., 1995.

[11] H. Gao, J. Lam, and C. Wang, "Robust energy-to-peak filter design for stochastic time-delay systems," Syst. Control Lett., vol. 55, no. 2, pp. 101-111, 2006.

[12] H. Gao, J. Lam, and C. Wang, "Induced $\boldsymbol{l}_{2}$ and generalized $\boldsymbol{H}_{2}$ filtering for systems with repeated scalar nonlinearities," IEEE Trans. Signal Processing, vol. 53, no. 11, pp. 4215-4226, Nov. 2005.

[13] T. Gardner, C. Cantor, and J. Collins, "Construction of a genetic toggle switch in Escherichia coli," Nature, vol. 403, pp. 339-342, 2000.

[14] S. Huang, "Gene expression profilling, genetic networks, and cellular states: An integrating concept for tumorigenesis and drug discovery," J. Molec. Med., vol. 77, pp. 469-480, 1999.

[15] M. de Hoon and S. Imoto et al., "Infering gene regulatory networks from time-ordered gene expression data of bacillus using differential equations," in Proc. Pacific Symp. Biocomput., 2003, vol. 8, pp. 17-28.

[16] J. Hasty, J. Pradines, M. Dolink, and J. Collins, "Biophysics noisebased switches and amplifiers for gene expression," Proc. Nat. Acad. Sci., vol. 97, no. 5, pp. 2075-2080, 2000.

[17] C. Li, L. Chen, and K. Aihara, "Synchronization of coupled nonidentical genetic oscillators," Phys. Biol., vol. 3, pp. 37-44, 2006.

[18] C. Li, L. Chen, and K. Aihara, "Stability of genetic networks with sum regulatory logic: Lur'e system and LMI approach," IEEE Trans. Circuits Syst. I, vol. 53, no. 11, pp. 2451-2458, Nov. 2006.

[19] C. Li, L. Chen, and K. Aihara, "Stochastic stability of genetic networks with disturbance attenuation," IEEE Trans. Circuits Syst. II, vol. 54, no. 10, pp. 892-896, Oct. 2007.

[20] C. Li, L. Chen, and K. Aihara, "Stochastic synchronization of genetic oscillator networks," BMC Syst. Biol., vol. 1, no. 6, pp. 1-11, 2007, 10.1186/1752-0509-1-6.

[21] H. Kushur, Stochastic Stability and Control. New York: Academic, 1976.

[22] X. Mao, Exponential Stability of Stochastic Differential Equations. New York: Marcel Dekker, 1994.

[23] N. A. M. Monk, "Oscillatory expression of Hes1, p53, and NF- $\kappa$ B driven by transcriptional time delays," Current Biol., vol. 13, pp. 1409-1413, 2003.

[24] J. Paulsson, "Summing up the noise in gene networks," Nature, vol. 427, pp. 415-418, 2004.

[25] A. Pease, D. Solas, E. Sullivan, M. Cronin, C. Holmes, and S. Fodor, "Light-generated oligonucleotide arrays for rapid DNA sequense analysis," Proc. Nat. Acad. Sci., vol. 91, pp. 5022-5026, 1994.
[26] F. Ren and J. Cao, "Asymptotic and robust stability of genetic regulatory networks with time-varying delays," Neurocomputing, vol. 71, no. 4-6, pp. 834-842, Jan. 2008.

[27] Z. Shu and J. Lam, "Delay-dependent exponential estimates of stochastic neural networks with time delay," in Proc. Int. Conf. Neural Inform. Processing, 2006, vol. 4232, pp. 332-341, Part I.

[28] P. Smolen, D. Baxter, and J. Byrne, "Mathematical modeling of gene networks," Neuron, vol. 26, pp. 567-580, 2000.

[29] P. Smolen, D. Baxter, and J. Byrne, "Modelling circadian oscillations with interlocking positive and negative feedback loops," J. Neurosci., vol. 21, pp. 6644-6656, 2001.

[30] T. Tian and K. Burrage, "Stochastic neural network models for gene regulatory networks," in Proc. IEEE Congress Evol. Computat., 2003, pp. $162-169$.

[31] Z. Wang, F. Yang, D. W. C. Ho, and X. Liu, "Robust $\boldsymbol{H}_{\infty}$ filtering for stochastic time-delay systems with missing measurements," IEEE Trans. Signal Processing, vol. 54, no. 7, pp. 2579-2587, Jul. 2006

[32] Z. Wang and K. Burnham, "Robust filtering for a class of stochastic uncertain nonlinear time-delay systems via exponential state estimation," IEEE Trans. Signal Processing, vol. 49, no. 4, pp. 794-804, Apr. 2001.

[33] Z. Wang, F. Yang, D. W. C. Ho, S. Swift, A. Tucker, and $\mathrm{X}$. Liu, "Stochastic dynamic modeling of short gene expression time series data," IEEE Trans. NanoBiosci., vol. 7, no. 1, pp. 44-55, Mar. 2008.

[34] Z. Wang, Y. Liu, and X. Liu, “ $\boldsymbol{H}_{\infty}$ filtering for uncertain stochastic time-delay systems with sector-bounded nonlinearities," Automatica, vol. 44, no. 5, pp. 1268-1277, 2008.

[35] G. Wei, Z. Wang, H. Shu, K. Fraser, and X. Liu, "Robust filtering for gene expression time series data with variance constraints," Int. J. Comput. Math., vol. 84, no. 5, pp. 619-633, 2007.

[36] B. Xu and Y. Tao, "External noise and feedback regulation: Steadystate statistics of auto-regulatory genetic network," J. Theor. Biol., vol. 243, pp. 214-221, 2006. 